\title{
Three-Dimensional Spatiotemporal Pulse-Train Solitons
}

\author{
Oren Lahav, ${ }^{1, *}$ Ofer Kfir, ${ }^{1,2}$ Pavel Sidorenko, ${ }^{1}$ Maor Mutzafi, ${ }^{1}$ Avner Fleischer,${ }^{1,3,4}$ and Oren Cohen ${ }^{1, \dagger}$ \\ ${ }^{1}$ Department of Physics and Solid State Institute, Technion, Haifa 32000, Israel \\ ${ }^{2}$ Physical Institute, Georg-August University of Göttingen, Göttingen 37007, Germany \\ ${ }^{3}$ Raymond and Beverly Sackler Faculty of Exact Sciences, School of Chemistry, \\ Tel Aviv University, Tel Aviv 6997801, Israel \\ ${ }^{4}$ Tel-Aviv University Center for Light-Matter-Interaction, Tel Aviv 6997801, Israel \\ (Received 10 July 2017; revised manuscript received 18 September 2017; published 30 November 2017)
}

Experimental realization of three-dimensional spatiotemporal solitons, which were proposed several decades ago, is still considered a "grand challenge" in nonlinear science. Here, we present experimental observation of 3D optical spatiotemporal pulse-train solitons. A spatially bright temporally dark pulse-train beam is trapped in a bulk medium that supports two types of nonlinearities: slowly responding saturable selffocusing that collectively self-trap the beam in the transverse directions and fast self-phase modulation that self-localizes each dark notch temporally (longitudinally). This work opens the possibility for experimental investigations of various soliton phenomena, including soliton interaction in $3 \mathrm{D}$, formation of multimode spatiotemporal solitons, and envisioning new entities like partially coherent spatiotemporal solitons.

DOI: 10.1103/PhysRevX.7.041051

Solitons are self-trapped wave packets for which nonlinearity robustly balances linear broadening and that exhibit particlelike properties [1,2]. Solitons that are self-trapped in one or two dimensions (in space or time) have been observed in many systems, including shallow [3] and deep [4] water waves, the nervous system [5], meteorology [6], 'Bose-Einstein condensates [7,8], and optics [9-12]. Threedimensional spatial solitons were only recently observed in chiral ferromagnetic liquid crystal colloids $[13,14]$.

In addition to spatial and temporal solitons, solitons can also be trapped in both space and time, simultaneously [15]. Since the proposal of optical spatiotemporal solitons (STS), almost three decades ago [16], they have evolved into a vibrant field of research that combines spatial, temporal, and nonlinear optics [17-20]. 3D STS, which are also termed "light bullets," have particularly been attracting substantial theoretical interest in both fundamental $[17,21,22]$ and applied science $[23,24]$. The experimental progress, on the other hand, has been quite limited. The main challenges include the matching of the diffraction and dispersion lengths, which should be balanced simultaneously by the nonlinearity, and stability to noise, which is especially challenging for high-dimensional solitons [16-20]. Spatiotemporal solitons

\footnotetext{
*Corresponding author. orenla@technion.ac.il

Corresponding author. oren@technion.ac.il

Published by the American Physical Society under the terms of the Creative Commons Attribution 4.0 International license. Further distribution of this work must maintain attribution to the author(s) and the published article's title, journal citation, and DOI.
}

Subject Areas: Nonlinear Dynamics,

Optics were first demonstrated experimentally in 1999 by employing the cascaded second harmonic generation process in bulk medium [25]. However, these solitons are fundamentally two dimensional; i.e., they are trapped in time and in only one spatial transverse direction while they are very wide and spectrally dispersed in the other transverse direction. The propagation distance of these solitons is limited by transverse instability. More recently, quasi-3D STS were experimentally demonstrated in an array of waveguides with Kerr nonlinearity [26,27]. These discrete light bullets (LBs) propagated with approximately stationary pulse width for about two diffraction lengths. The higher-order nonlinear effects, that lead to spectral redshift, upsets the balance between the wavelength-sensitive discrete diffraction and self-focusing effects and eventually results in the disintegration of these LBs. Early this year, mode-locked spatial cavity solitons with pulse duration shorter than the cavity round-trip were observed [28]. This research direction may enable generation of bright 3D cavity solitons. Linear light bullets employing the nondiffraction property of Airy and Bessel beams were demonstrated [29,30]. Still, stable 3D STS (that can support solitons interactions) have not been experimentally demonstrated. Whilst in optical lattices quasi 3D STS were observed, no similar progress has been made in homogeneous media.

In 2009, spatiotemporal pulse-train solitons (STPTSs) were proposed, offering a new path toward generation of 3D STS [31,32]. A STPTS consists of a sequence of short pulses that are collectively trapped in the transverse directions by a slow self-focusing mechanism and, simultaneously, each pulse is self-trapped temporally by a fast self-phase modulation (e.g., induced by the Kerr nonlinearity). The response time of the slow self-focusing is 


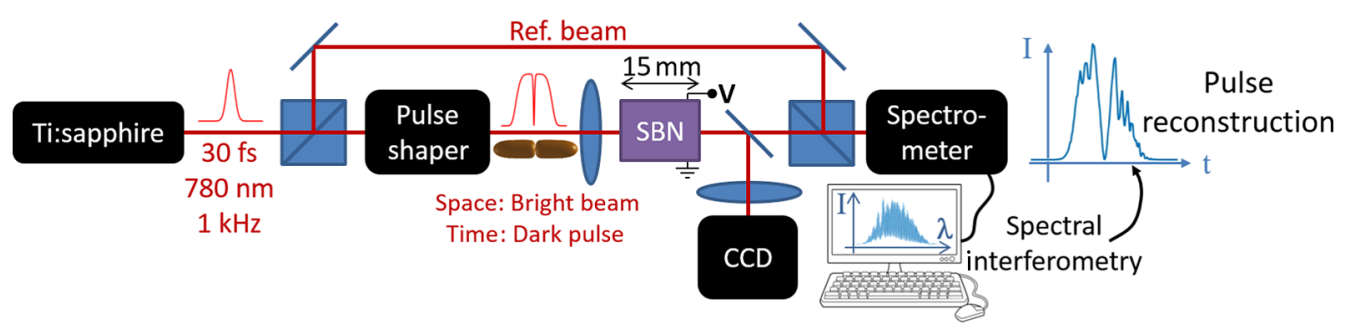

FIG. 1. Experimental setup. A Ti:sapphire laser pulse-train beam $(\lambda=0.8 \mu \mathrm{m})$ with $1-\mathrm{KHz}$ repetition rate and 30-fs pulse duration is divided into reference and signal beams. The signal beam propagates through a pulse shaper designed to form a dark pulse (100-fs FWHM) centered at a relatively wide background pulse (1-ps FWHM). The dark pulse-train beam is then focused to the input face of a photorefractive SBN crystal $(5 \times 5 \times 15 \mathrm{~mm}$ and $6 \times 6 \times 6 \mathrm{~mm})$. The spatial intensity distributions at the input and output faces of the crystal are recorded by an imaging system and CCD. The temporal pulse profile is characterized through spectral interferometry by interfering the reference and signal beams in a spectrometer.

much longer than the time interval between consecutive pulses, hence, it is affected by the averaged power of the beam; i.e., it is insensitive to the temporal profile of the pulses. The characteristic lengths of the slow and fast nonlinearities are tuned to match the diffraction and dispersion lengths, respectively. For stability, the slow self-focusing should be nonlocal or saturable and significantly larger than the fast nonlinearity. Two types of STPTSs were predicted in Refs. [31,32]. In one type, the STPTSs are bright (i.e., their profiles are bell-like) in all directions, while in the other type they are dark in the time domain (a dark soliton is a self-localized intensity dip embedded in a continuous background [33]). Fully bright STPTSs can exist in anomalous dispersive media while spatially bright temporally dark STPTSs require normal dispersion. Following Refs. [31,32], the twononlinearities approach was employed for predicting generation of dense LB trains [34] and experimental demonstrations of third-harmonic generation [35] and spectral broadening [36] within 2D spatial solitons.
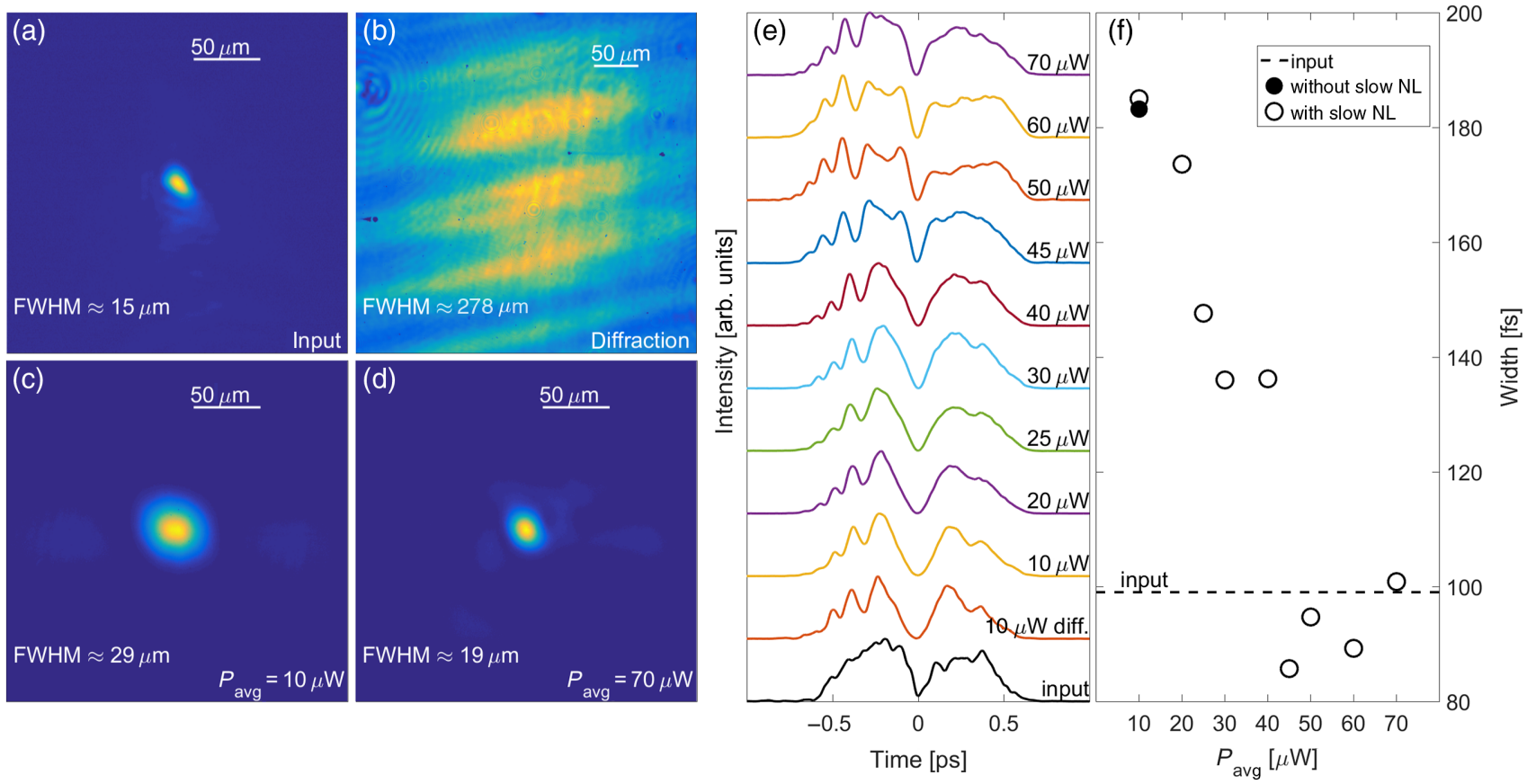

FIG. 2. Three-dimensional spatiotemporal pulse-train soliton in a long crystal $(L=15 \mathrm{~mm})$. (a) Spatial intensity distribution at the input plane of the crystal (FWHM $\approx 15 \mu \mathrm{m}$ ). (b) The intensity distribution at the output plane of the crystal under approximately linear propagation condition (the screening self-focusing is turned off by applying zero external voltage and the Kerr nonlinearity is small because the intensity of the beam is low- $-10 \mu \mathrm{W}$ averaged power of the incoming beam). The beam broadens to FWHM $\approx 278 \mu \mathrm{m}$. Output plane intensity distributions when the screening self-focusing is turned on $(V=500 \mathrm{~V})$ at (c) $10 \mu \mathrm{W}$ and (d) $70 \mu \mathrm{W}$ averaged powers, demonstrating the transverse spatial self-trapping (FWHM $\approx 29 \mu \mathrm{m}$ and FWHM $\approx 19 \mu \mathrm{m}$, respectively). (e) Reconstructed intensity pulse profiles of (bottom up) input pulse (black), diffracted beam with $P_{\text {avg }}=10 \mu \mathrm{W}$ (red), and spatially trapped beam with $P_{\text {avg }}=10,20,25,30,40,45,50,60$, and $70 \mu \mathrm{W}$. (f) The duration of the dark notch as a function of average power demonstrating the temporal confinement. The horizontal dashed line corresponds to the duration at the input plane. 

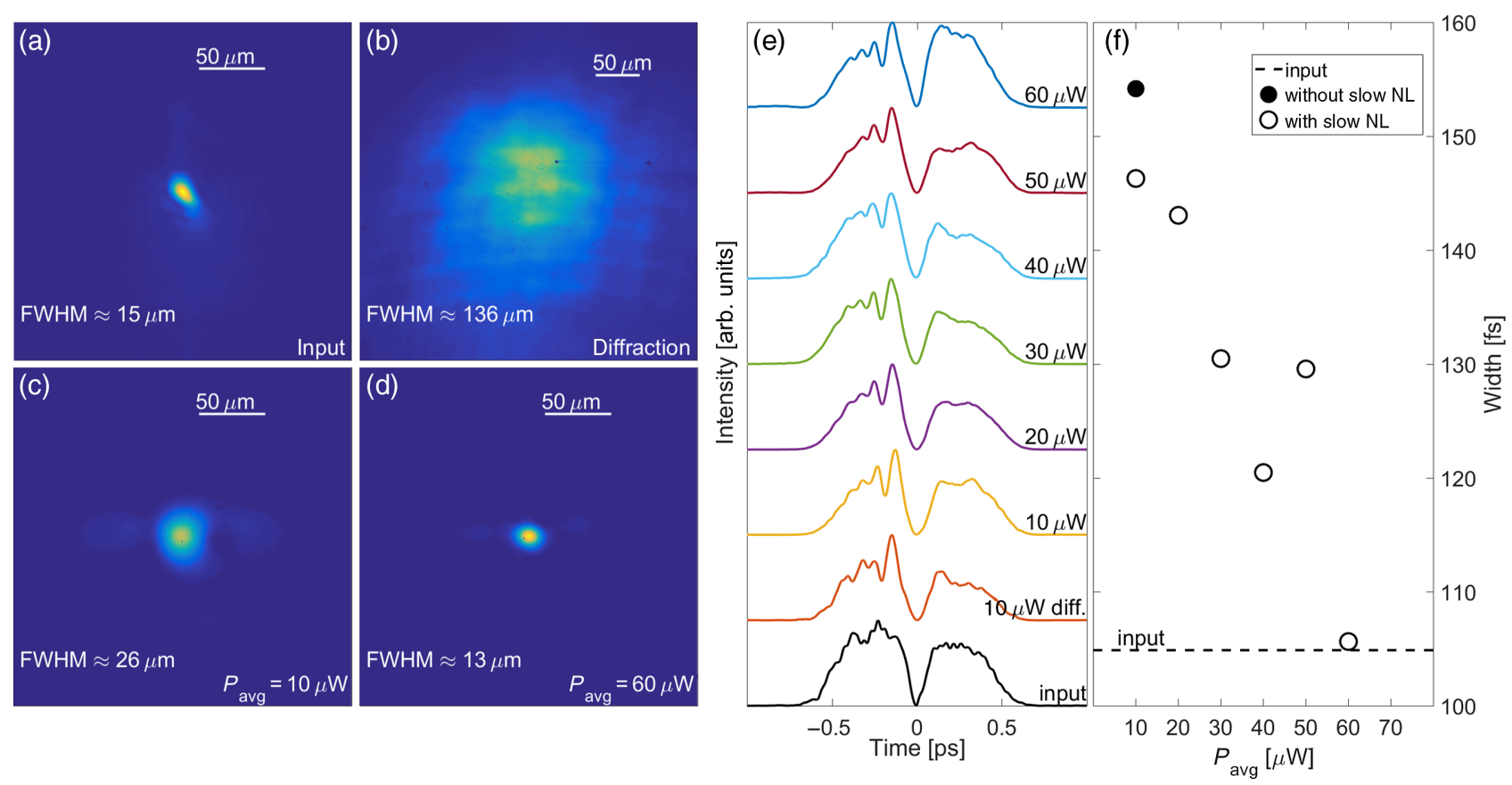

FIG. 3. Three-dimensional spatiotemporal pulse-train soliton in a short crystal $(L=6 \mathrm{~mm})$. (a) Spatial intensity distribution at the input plane of the crystal (FWHM $\approx 15 \mu \mathrm{m})$. (b) The intensity distribution at the output plane of the crystal under approximately linear propagation condition. The beam broadens to FWHM $\approx 136 \mu \mathrm{m}$. Output plane intensity distributions when the screening self-focusing is turned on $(V=1800 \mathrm{~V}$ ) at (c) $10 \mu \mathrm{W}$ and (d) $60 \mu \mathrm{W}$ averaged powers, demonstrating the transverse spatial self-trapping (FWHM $\approx 26 \mu \mathrm{m}$ and FWHM $\approx 13 \mu \mathrm{m}$, respectively). (e) Reconstructed intensity pulse profiles of (bottom up) input pulse (black), diffracted beam with $P_{\text {avg }}=10 \mu \mathrm{W}$ (red), and spatially trapped beam with $P_{\text {avg }}=10,20,30,40,50$, and $60 \mu \mathrm{W}$. (f) The duration of the dark notch as a function of average power demonstrating the temporal confinement. The horizontal dashed line corresponds to the duration at the input plane.

Here, we demonstrate experimentally 3D spatiotemporal pulse-train solitons. We observe solitonic propagation for 29 diffraction lengths and approximately two dispersion lengths, limited by the finite length of our crystals. Thus, investigations of 3D soliton interactions and other new phenomena should be accessible in this system. Our approach for experimentally generating STPTSs is to utilize the screening self-focusing in photorefractives [37]. This self-focusing is highly saturable; hence, it can support high-dimensional solitons [2] and is also very slow-its response time can be many seconds and even minutes, a feature that is utilized for generation of incoherent solitons [2]. Specifically, we use a strontium barium niobate (SBN) photorefractive crystal that was shown to support 2D spatial solitons of picosecond [38] and femtosecond [36] pulse-train beams at a wavelength of $0.8 \mu \mathrm{m}$. The dispersion of SBN is normal in this wavelength; hence, we generate here spatially bright temporally dark STPTSs.

The experimental setup is shown in Fig. 1. A Ti: sapphire laser beam with $1-\mathrm{KHz}$ repetition rate and 30 -fs pulse duration is divided into reference and signal beams. The signal beam propagates through a pulse shaper designed to form a dark pulse whose full width at half of the maximum intensity is $100 \mathrm{fs}$ centered at a relatively wide background pulse (a super-Gaussian with 1 ps FWHM). The pulse-train beam is then focused on the input face of a photorefractive SBN crystal $(5 \times 5 \times 15 \mathrm{~mm}$ and $6 \times 6 \times 6 \mathrm{~mm}$, as shown in Figs. 2 and 3 , respectively). The spatial intensity distributions at the input and output faces of the crystal are recorded by an imaging system and a CCD. The temporal pulse profile is characterized by spectral interferometry [39] (the reference pulse is characterized by using a frequency-resolved optical gating apparatus [40]).

The strength of the slow photorefractive nonlinearity is controlled via an applied voltage along the $c$ axis of the crystal, which is perpendicular to the propagation direction. The transverse self-trapping is demonstrated in Figs. 2(a)-2(d). Figure 2(a) shows the intensity distribution of the focused beam at the input face of the crystal $(\mathrm{FWHM} \approx 15 \mu \mathrm{m})$. When the photorefractive nonlinearity is off (zero applied voltage), the beam diffracts significantly after propagating along $15 \mathrm{~mm}$ in the SBN crystal [Fig. 2(b)] and reaches a size of FWHM $\approx 278 \mu \mathrm{m}$ (the observed modulation in the diffracted beam is due to growth-induced "striations" in the crystal [41]). When applying a high voltage $(500 \mathrm{~V})$ on the SBN crystal, a spatial self-trapping is achieved [36-38]. Figures 2(c) and 2(d) show the output trapped beam for an average power of 10 and $70 \mu \mathrm{W}$, respectively (we do not increase the averaged power beyond $70 \mu \mathrm{W}$ in order to not damage the crystal). The temporal self-trapping is presented in 
Figs. 2(e) and 2(f). Figure 2(e) shows the reconstructed intensity profiles of the input pulse (lowest curve) and the output pulses at various conditions, starting with an average power of $10 \mu \mathrm{W}$ and no photorefractive nonlinearity $(V=0)$, and then the output pulses with increasing averaged power with the photorefractive nonlinearity turned on. As shown in Fig. 2(f), the duration of the dark notch (determined according to the algorithm for calculating pulse duration described in Sec. 5.4.1 of Ref. [42]) decreases (as the power increases) from a linearly dispersed pulse of $185 \mathrm{fs}$ to the initial input pulse width of $100 \mathrm{fs}$. This 3D STS propagates for a distance of $15 \mathrm{~mm}$, which corresponds to 29 diffraction lengths and approximately 1.5 dispersion lengths. Such a long propagation distance should make the experimental investigations of collisions and interactions between 3D solitons accessible.

We repeat the measurements with a shorter crystal $(6 \times 6 \times 6 \mathrm{~mm})$ to verify that the $3 \mathrm{D}$ soliton wave packet is stationary along the propagation distance. The experimental results are shown in Fig. 3, which has the same structure as Fig. 2. Indeed, it shows that 3D spatiotemporal solitons are formed in the short and long crystals at similar parameters.

In conclusion, we demonstrate experimentally stable 3D spatiotemporal solitons. The experiment is based on utilizing a combination of slow and fast nonlinearities to produce spatiotemporal pulse-train solitons. It should now be possible to explore interactions and collisions between 3D solitons experimentally, some of which are predicted to be unique to 3D solitons [43]. Moreover, new phenomena are expected since STPTSs require both slow and fast nonlinearities. For example, consider interaction between STPTSs that overlap spatially. It will be interesting to explore this interaction as a function of the degree of temporal overlap between the pulses, from full overlap to absent temporal overlap. Also, it should be possible to produce multimode [44] and maybe even partially coherent spatiotemporal pulse-train solitons. An exciting next step is to produce a fully bright 3D STPTS, e.g., by utilizing the slowly responding, nonlocal thermal nonlinearity of fused silica at a spectral region near $2 \mu \mathrm{m}$, where the dispersion is anomalous [31,32]. Another promising research direction is to exploit the concept of spatiotemporal pulse-train solitons, where the temporal and spatial confinements are largely controlled by different knobs, for improving mode locking of lasers. All in all, we expect that the new experimental platform and high dimensionality will give rise to fundamental and applicable new opportunities in soliton science.

\section{ACKNOWLEDGMENTS}

We would like to thank the support of the Israeli Center of Research Excellence 'Circle of Light' (1802/12), The Wolfson Foundation, and German Israeli Foundation (GIF).
[1] T. Dauxois and M. Peyrard, Physics of Solitons (Cambridge University Press, Cambridge, England, 2006).

[2] Z. Chen, M. Segev, and D. N. Christodoulides, Optical Spatial Solitons: Historical Overview and Recent Advances, Rep. Prog. Phys. 75, 086401 (2012).

[3] J. S. Russell, Report of the Fourteenth Meeting of the British Association for the Advancement of Science, York, 1844 (John Murray, London 1845), pp. 311-390.

[4] C. R. Jackson, An Atlas of Internal Solitary-like Waves and Their Properties, 2nd ed. (Global Ocean Associates, Alexandria, 2004).

[5] A. Gonzalez-Perez, R. Budvytyte, L. D. Mosgaard, S. Nissen, and T. Heimburg, Penetration of Action Potentials During Collision in the Median and Lateral Giant Axons of Invertebrates, Phys. Rev. X 4, 031047 (2014).

[6] R. H. Clarke, R. K. Smith, and D. G. Reid, The Morning Glory of the Gulf of Carpentaria: An Atmospheric Undular Bore, Mon. Weather Rev. 109, 1726 (1981).

[7] K. E. Strecker, G. B. Partridge, A. G. Truscott, and R. G. Hulet, Formation and Propagation of Matter-Wave Soliton Trains, Nature (London) 417, 150 (2002).

[8] L. Khaykovich, F. Schreck, G. Ferrari, T. Bourdel, J. Cubizolles, L. D. Carr, Y. Castin, and C. Salomon, Formation of a Matter-Wave Bright Soliton, Science 296, 1290 (2002).

[9] J. E. Bjorkholm and A. A. Ashkin, cw Self-Focusing and Self-Trapping of Light in Sodium Vapor, Phys. Rev. Lett. 32, 129 (1974).

[10] L. F. Mollenauer, R. H. Stolen, and J. P. Gordon, Experimental Observation of Picosecond Pulse Narrowing and Solitons in Optical Fibers, Phys. Rev. Lett. 45, 1095 (1980).

[11] A. Barthelemy, S. Maneuf, and C. Froehly, Propagation Soliton et Auto-Confinement de Faisceaux Laser par Non Linearité Optique de Kerr, Opt. Commun. 55, 201 (1985).

[12] Y.S. Kivshar and G. P. Agrawal, Optical Solitons: From Fibers to Photonic Crystals (Academic Press, New York, 2003).

[13] P. J. Ackerman and I. I. Smalyukh, Static Three-Dimensional Topological Solitons in Fluid Chiral Ferromagnets and Colloids, Nat. Mater. 16, 426 (2017).

[14] P. J. Ackerman and I. I. Smalyukh, Diversity of Knot Solitons in Liquid Crystals Manifested by Linking of Preimages in Torons and Hopfions, Phys. Rev. X 7, 011006 (2017).

[15] V. E. Zakharov and E. A. KuznetsovZh. Eksp. Teor. Fiz. 66, 594 (1974) [Three-Dimensional Solitons, Sov. Phys. JETP 39, 285 (1974)].

[16] Y. Silberberg, Collapse of Optical Pulses, Opt. Lett. 15, 1282 (1990).

[17] F. W. Wise and P. Di Trapani, The Hunt for Light Bullets-Spatiotemporal Solitons, Opt. Photonics News 13, 28 (2002).

[18] B. A. Malomed, D. Mihalache, F. W. Wise, and L. Torner, Spatiotemporal Optical Solitons, J. Opt. B 7, R53 (2005).

[19] D. Mihalache, Multidimensional Localized Structures in Optics and Bose-Einstein Condensates: A Selection of Recent Studies, Rom. J. Phys. 59, 295 (2014).

[20] B. A. Malomed, Multidimensional Solitons: WellEstablished Results and Novel Findings, Eur. Phys. J. Spec. Top. 225, 2507 (2016). 
[21] G. H. Derrick, Comments on Nonlinear Wave Equations as Models for Elementary Particles, J. Math. Phys. 5, 1252 (1964).

[22] A. A. Kanashov and A. M. Rubenchik, On Diffraction and Dispersion Effect on Three Wave Interaction, Physica (Amsterdam) 4D, 122 (1981).

[23] R. McLeod, K. Wagner, and S. Blair, $(3+1)$-Dimensional Optical Soliton Dragging Logic, Phys. Rev. A 52, 3254 (1995).

[24] M. O. Williams, C. W. McGrath, and J. N. Kutz, LightBullet Routing and Control with Planar Waveguide Arrays, Opt. Express 18, 11671 (2010).

[25] X. Liu, L. J. Qian, and F. W. Wise, Generation of Optical Spatiotemporal Solitons, Phys. Rev. Lett. 82, 4631 (1999).

[26] S. Minardi, F. Eilenberger, Y. V. Kartashov, A. Szameit, U. Röpke, J. Kobelke, K. Schuster, H. Bartelt, S. Nolte, L. Torner, F. Lederer, A. Tünnermann, and T. Pertsch, Three-Dimensional Light Bullets in Arrays of Waveguides, Phys. Rev. Lett. 105, 263901 (2010).

[27] F. Eilenberger, K. Prater, S. Minardi, R. Geiss, U. Röpke, J. Kobelke, K. Schuster, H. Bartelt, S. Nolte, A. Tünnermann, and T. Pertsch, Observation of Discrete, Vortex Light Bullets, Phys. Rev. X 3, 041031 (2013).

[28] F. Gustave, N. Radwell, C. McIntyre, J. P. Toomey, D. M. Kane, S. Barland, W. J. Firth, G.-L. Oppo, and T. Ackemann, Observation of Mode-Locked Spatial Laser Solitons, Phys. Rev. Lett. 118, 044102 (2017).

[29] A. Chong, W. H. Renninger, D. N. Christodoulides, and F. W. Wise, Airy-Bessel Wave Packets as Versatile Linear Light Bullets, Nat. Photonics 4, 103 (2010).

[30] D. Abdollahpour, S. Suntsov, D. G. Papazoglou, and S. Tzortzakis, Spatiotemporal Airy Light Bullets in the Linear and Nonlinear Regimes, Phys. Rev. Lett. 105, 253901 (2010).

[31] H. C. Gurgov and O. Cohen, Spatiotemporal Pulse-Train Solitons, Opt. Express 17, 7052 (2009).

[32] I. B. Burgess, M. Peccianti, G. Assanto, and R. Morandotti, Accessible Light Bullets via Synergetic Nonlinearities, Phys. Rev. Lett. 102, 203903 (2009).
[33] Y. S. Kivshar and B. Luther-davies, Dark Optical Solitons: Physics and Applications, Phys. Rep. 298, 81 (1998).

[34] M. Peccianti, I. B. Burgess, G. Assanto, and R. Morandotti, Space-Time Bullet Trains via Modulation Instability and Nonlocal Solitons., Opt. Express 18, 5934 (2010).

[35] M. Peccianti, A. Pasquazi, G. Assanto, and R. Morandotti, Enhancement of Third-Harmonic Generation in Nonlocal Spatial Solitons, Opt. Lett. 35, 3342 (2010).

[36] O. Lahav, H. Gurgov, P. Sidorenko, O. Peleg, L. Levi, A. Fleischer, and O. Cohen, Self-Phase Modulation Spectral Broadening in Two-Dimensional Spatial Solitons: Toward Three-Dimensional Spatiotemporal Pulse-Train Solitons, Opt. Lett. 37, 5196 (2012).

[37] M. Shih, P. Leach, M. Segev, M. H. Garrett, G. J. Salamo, and G.C. Valley, Two-Dimensional Steady-State Photorefractive Screening Solitons, Opt. Lett. 21, 324 (1996).

[38] J. Imbrock, C. Heese, and C. Denz, Spatial Photorefractive Solitons with Picosecond Laser Pulses, Appl. Phys. B 95, 261 (2009).

[39] C. Iaconis and I. A. Walmsley, Self-Referencing Spectral Interferometry for Measuring Ultrashort Optical Pulses, IEEE J. Quantum Electron. 35, 501 (1999).

[40] K. W. DeLong, R. Trebino, J. Hunter, and W. E. White, Frequency-Resolved Optical Gating with the Use of Second-Harmonic Generation, J. Opt. Soc. Am. B 11, 2206 (1994).

[41] K. Bastwöste, U. Sander, and M. Imlau, Conical Light Scattering in Strontium Barium Niobate Crystals Related to an Intrinsic Composition Inhomogeneity, J. Phys. Condens. Matter 19, 156225 (2007).

[42] IEEE® Standard on Transitions, Pulses, and Related Waveforms (IEEE, New York, 2011), http://ieeexplore .ieee.org/servlet/opac?punumber $=6016196$.

[43] D. E. Edmundson and R. H. Enns, Fully Three-Dimensional Collisions of Bistable Light Bullets, Opt. Lett. 18, 1609 (1993).

[44] L. G. Wright, W. H. Renninger, D. N. Christodoulides, and F. W. Wise, Spatiotemporal Dynamics of Multimode Optical Solitons, Opt. Express 23, 3492 (2015). 